\title{
Odd versus even: a scientific study of the 'rules' of plating
}

Andy T Woods, Charles Michel, Charles Spence

We report on the results of a series of large-scale computer-based preference tests (conducted at The Science Museum in London and online) that evaluated the widely-held belief that food should be plated in odd rather than even numbers of elements in order to maximize the eye appeal of a dish. Participants were presented with pairs of plates of food showing odd versus even number of seared scallops (3 vs. 4; 1-6 in Experiment 7), arranged in a line, as a polygon or randomly, on either a round or square white plate. No consistent evidence for a preference for odd or even number of food items was found, thus questioning the oft-made assertion that odd number of items on a plate looks better than an even numbers. The implications of these results are discussed. 
2 RUNNING HEAD: ODD VERSUS EVEN: A SCIENTIFIC STUDY OF THE 'RULES' OF 3 PLATING

Odd versus even?

An investigation on the impact of number of food items on plating preferences

Andy T. Woods, Charles Michel, \& Charles Spence

10

DATE: NOVEMBER 2015

17 RESUBMITTED TO: PeerJ

WORD COUNT: 13,056 WORDS

19

CORRESPONDENCE TO: Andy Woods, Crossmodal Research Laboratory, Department of 21 Experimental Psychology, University of Oxford, Oxford OX1 3UD, UK; E-MAIL: 22 andytwoods@gmail.com, TEL: +44 1865 271307; FAX: +44 1865310447. 
26 We report on the results of a series of large-scale computer-based preference tests (conducted at

27 The Science Museum in London and online) that evaluated the widely-held belief that food should 28 be plated in odd rather than even numbers of elements in order to maximize the eye appeal of a 29 dish. Participants were presented with pairs of plates of food showing odd versus even number of 30 seared scallops (3 vs. 4; 1-6 in Experiment 7), arranged in a line, as a polygon or randomly, on 31 either a round or square white plate. No consistent evidence for a preference for odd or even 32 number of food items was found, thus questioning the oft-made assertion that odd number of items 33 on a plate looks better than an even numbers. The implications of these results are discussed. 

— all good things come in threes not four (popular German saying).

43 Disciplines that involve arranging items to maximize their aesthetic appeal share the common aim 44 of seducing the eyes of the observer. From gardeners to chefs - plants and rocks at one end, to food elements on the plate on the other-each discipline has its own insights concerning ways to enhance composition. These ideas are often transmitted orally, or sometimes, via books or guides. We believe that while the approach has historically been driven by experience, studying which of those guidelines are effective (and which of those that turn out to be effective cross-culturally) could benefit both these artisanal (or, may we say, artistic) disciplines, but also pose interesting scientific questions as to the nature of those biases, should they be confirmed empirically.

One such guideline is the belief that it is better to present odd rather than even numbers of items (e.g., Van Tonder \& Lyons, 2005)1. But is this anything more than 'an old wives' tale? Chefs often acknowledge the importance of presenting odd numbers of elements on the plate, as recommended in chefs' guides on the art of plating (e.g., Styler \& Lazarus, 2006), in an attempt to enhance the visual appeal of a meal ${ }^{2}$. However, to the best of our knowledge, this claim has not been empirically tested previously. So the question that we wish to address here is: "Do odd vs. even numbers really matter when it comes to the visual appreciation of compositions?" And, to what extent can this difference influence the visual appreciation of the food, or maybe even the actual enjoyment of the food? One way of testing whether an odd number of items on a plate is preferred over an even number is to show participants two such plates of food, and ask them which they

\footnotetext{
${ }^{1}$ The idea of a preference for odd rather than even numbers of elements is not new. In the traditional art of Japanese rock gardens, for instance, ancient texts mention the importance of preferring odd vs. even numbers (Shimoyama, 1976; Van Tonder \& Lyons, 2005), not only in terms of odd-numbered groups of rocks, but also that the different clusters of rocks should also be an odd-number.

2 An eagle-eyed reviewer raised the possibility that an odd number of food-items could be preferred over an even number due to reasons of etiquette. Their example was that, "given the assumption that food on a dish might be consumed only partially (and this would very much apply to a context of cuisine rather than kitchen), an odd-item presentation would favor a division with remainders?"
} 
61 prefer. Unfortunately, any two such plates of food would undoubtedly differ in more ways than 62 just the number of items that they contain, which makes it hard to tease out the underlying driver 63 of liking. We discuss these issues shortly.

64 In terms of food, there is very little research on the topic. Furthermore, none of this research has 65 focused on the question of odd versus even numbers. Bajaj was one of the only researchers to 66 tangentially address this issue. In his doctoral thesis, 215 participants were given the option of eating a piece of chicken cut into either 4 pieces, or left as a single piece (Bajaj, 2013, Chapter 3 Experiment 1). Although significantly more participants chose the 4-item dish over the 1-item dish than could be expected by chance $(148, \mathrm{p}<.001)$, no difference in pleasantness was reported between these individuals and those deciding on the 1-item dish. In a second study, 301 participants were randomly assigned to meal type (a bagel served in 4 pieces vs. whole) but pleasantness did not vary across the groups of participants. The issue with these studies, in relation to 'odd versus even' number of items on the plate, is that the number of food items were quite different (1 vs. 4). We would expect, and will discuss next, a range of issues that might have swayed one's opinion on dish preference, which most likely are only exacerbated by large differences in the number of items / sub-portions.

For example, Geier, Rozin, and Doros (2006) put forward, and subsequently demonstrated, the concept of 'unit bias', where when given the option to eat to satiation items of a small or large size, much smaller quantities of the small items were consumed than of the large. The consequence could be that, when asked to choose between plates of food, the most appetising portion will be that which matches one's current level of hunger (or dieting ambitions; see Forde, Almiron-Roig, \& Brunstom, 2015, for a recent review on expected sensation in food selection). The logical consequence for preference between odd versus even number of items on a plate is that, if one portion appears larger than the other, this will have a knock-on effect on choice selection.

However, even if portions are equated in terms of their calorific content, a variety of phenomena can act to influence just how large a given portion of food may seem. For example, the size of the plate in relation to the food it contains has also been shown to influence perceived portion size thanks to the Delboeuf illusion (see McClain et al., 2014; Spence, Piqueras-Fiszman, Michel, \& 
90 as larger than they actually are when there is a small size difference between the circles, but smaller

91 than they actually are if the size difference in larger.

92 The visual balance of the composition can influence how we perceive and how much we like food 93 (for an overview, see Spence, et al., 2014, Michel, Velasco, Woods, \& Spence, submitted) and can 94 presumably influence whether odd or even number of items on the plate are preferred. In terms of 95 balance, Zellner et al. (2011, p. 642) states that: "The presentation of a plate offood can be thought 96 of as 'balanced' if that plate of food looks like it would balance when placed on a narrow central 97 pedestal. That is, the food is distributed in a manner around the central point such that the 98 perceived heaviness in one area looks balanced by equal heaviness on the opposite side of the 99 plate." Zellner et al. (2010) found that balance, in conjunction with food colour (or lack of it), 100 influenced attractiveness of the visual presentation.

101 The artistic principles of visual harmony, including balance, contrast, emphasis, pattern, 102 proportion, rhythm, unity, and variety (Arnheim, 1988; Bouleau, 1980; Wilson \& Chatterjee, 103 2005), could also influence food preference (Spence et al., 2014). Some aspect of harmony could 104 help to determine whether one prefers an odd versus an even number of items on the plate. Indeed, 105 muddying the issue somewhat, the plate on which the food is presented could itself play in 106 important role (as the 'frame' of the food).

108 Overview

109 We report on a series of experiments that are currently running at the Science Museum in London 110 (see citizen science experiment). Participants were presented with photos of pairs of plates of food 111 and asked to choose which one they preferred. The pairs always consisted of individual dishes of

112 food, one containing an even number of seared scallops and the other an odd number of the same 113 food. We also assessed any interaction between odd/even, arrangement of the elements (line vs. 114 polygon), and the shape of the plate on which the food elements happened to be presented.

115 The results of our first study revealed an intriguing interaction between odd/even and the shape of 116 the plate on which the elements were arranged. There was, however no consistent evidence for our 117 hypothesis that 3-items would be preferred to 4-items though. We explored these effects over a 
118 series of follow-up studies conducted online through Amazon's Mechanical Turk (MTurk). We

119 controlled for the effects of crowding on the plate (Experiment 2), we equated portion size across

120 the dishes (Experiments 3 and two further experiments reported as Supplementary materials), and

121 we also tested for effects of portion size distortion (Experiment 4). The results of a Combined

122 Analysis revealed that it was portion size that was the driving factor for both the participants at the

123 Science Museum and those recruited via MTurk. These two groups of participants differed,

124 though, in terms of which dish (odd versus even) they preferred when portion size was equated

125 over plates. Whilst the participants in the Science Museum study appeared to prefer 3-items at this

126 'equal portion-size' point, the MTurk participants preferred 4. In Experiment 5, we ruled out the

127 possibility that this difference was attributable to a small difference in the overall size of the two

128 portions. We tested a third group of participants recruited through Prolific Academic in

129 Experiment 6 to determine whether this group would have yet another equal portion size-point,

130 but this was not the case. That is, the values obtained from this group did not really differ from

131 that of MTurk participants. We argue, though, that the ratio-effect most likely arises due to some

132 difference in the characteristics of the populations tested. In Experiment 7 we tested plates

133 containing a range of number of elements, all of which though were of the same portion size, and

134 found that generally, plates with more elements were preferred over those with fewer elements.

135 Whether the dishes contained an odd or even number of elements played no role in this finding.

\section{EXPERIMENT 1}

138 Here we tested the hypothesis that participants would prefer a dish of food containing 3-items of

139 food over one containing 4-items.

MATERIALS AND METHODS

\section{Participants}

1421816 individuals (1305 female and 509 male; 2 did not report whether they were male or female)

143 took part in a citizen science experiment, conducted at the Science Museum in London during

144 February to April 2015. The experiment could either be performed online (598 individuals) ${ }^{3}$ or in 
145 an interactive digital platform at the 'Antenna Gallery', as part of an exhibition on the science of 146 eating called 'Cravings'. The online participants were invited to access this experiment via the 147 information page of 'Cravings' exhibition, and from the Science Museum's home webpage. At the 148 museum's gallery, the digital platform was one of the attractions of the exhibition.

149 The median age of the participants was in the 16-34 years range (note that the participants specified 150 if there age was $<16,16-34,35-54,55-74$ or $75+$; the respective counts in each group were 447 , $151880,383,92$ and 12; 2 people did not report their age). All of the participants were informed about

152 the nature of the study, and provided informed consent prior to taking part in the study and all of 153 the studies reported thereafter. These studies have been approved by Oxford University's Medical 154 Sciences Inter-Divisional Research Ethics Committee (approval \# MSD-IDREC-C1-2015-004).

\section{Stimuli}

157 Scallops were chosen for the study, given that they are similar in shape (round) and size. Fresh 158 scallops were seared in a hot pan with butter, in order to attain a light brown colouring. The same 159 set of scallops was then placed and photographed on a white surface. Note that the scallops were 160 photographed from a zenithal perspective with zenithal lighting, in order to avoid any shadow on 161 the food. The scallop images were then cut and placed digitally on the different plates (square or 162 round, photographed in the same way as the scallops). The stimuli used in this experiment are 163 shown in Figure 1.

3 This experiment runs from the 20th of February 2015, until January 2016, see http://bit.ly/1MwGh35 to access the online experiment. 


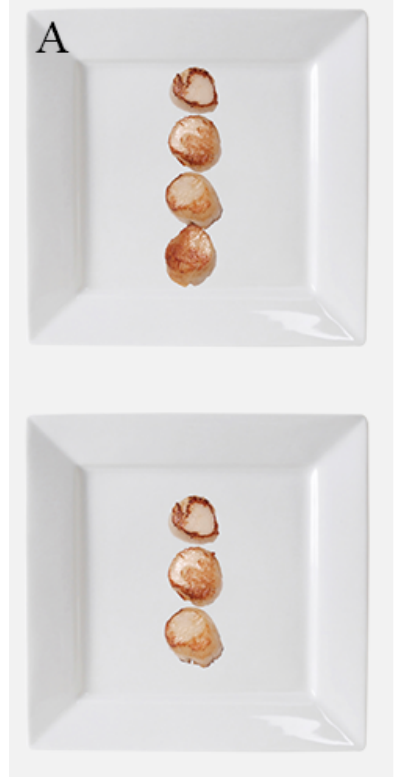

$\mathrm{B}$

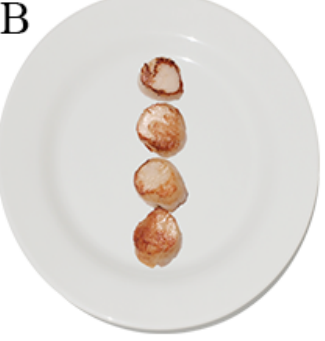

$\mathrm{C}$

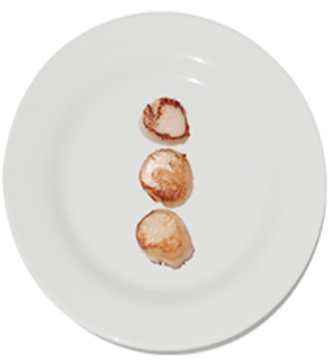

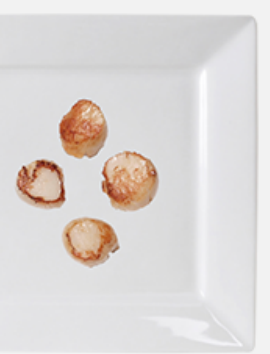

$\mathrm{D}$
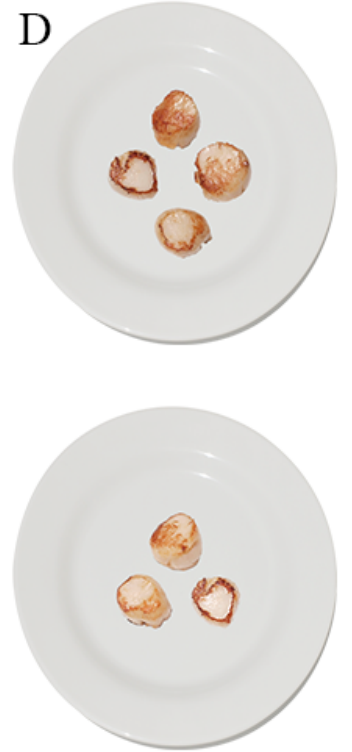

Figure 1. The 8 plates of scallops that were presented to the participants in Experiment 1. The plates were presented in pairs (specifically, the upper and lower image in each column was compared). The plates vary systematically in terms of the number of seared scallops ( 3 vs. 4), the arrangement of the scallops (line vs. polygon), and the shape of

168 the plate (round vs. angular).

\section{Design}

171 The dependent variable was the preferred dish chosen by the participants.

\section{Procedure}

173 The participants who took part in this experiment undertook five or more different tasks. The order

174 in which the tasks were presented and the different conditions was randomised, as were the left or 175 rightward position of the dishes. In the experiments reported here, 164 participants undertook two 176 trials whilst the remainder completed only a single trial. The participants could either submit their

177 answer by clicking on a circular button placed right below the food image, leave the experiment 178 by clicking on an ' $\mathrm{X}$ ' button, or go on to the next question by clicking on the 'Skip' button (see 179 Figure 2). 


\section{Which plate of food do you like more?}

180
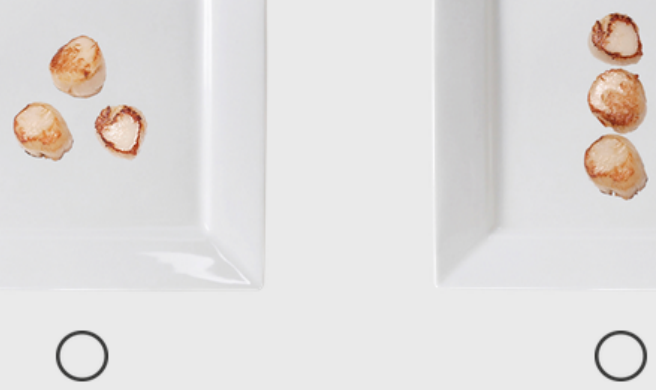

$\times$

Skip

181 Figure 2. The arrangement of the scallops shown to participants on a single trial.

182

183

\section{RESULTS}

184 The results, split by condition, are shown in Figure 3. A log-linear analysis was performed, using

185 Plate Shape (circular, square) x food Arrangement (vertical, polygonal) x food Items $(3,4)$ as the variables (the final model's likelihood ratio was $\chi^{2}(2)=3.27, p=.20$ ). The Arrangement $\mathrm{x}$ Items $\chi^{2}(1)=54.84, p<.001$, and Plate $x$ Items interactions were retained by the model, $\chi^{2}(1)=6.63, p=.01$.

188 Both interactions were explored by means of separate follow-up tests designed to assess whether 3 vs. 4 Items differed over the levels of the interacting factor.

190 In terms of the first interaction, in line with our hypothesis, 3 items that were arranged vertically were 1.24 times more likely to be chosen that 4 vertically arranged items $(p<.001$; with 428 picking

192 the 4 item dish and 531 picking the 3 item dish; 95\% CI 52.16\%, 58.55\%). Contrary to the

193 hypothesis however, 4 items arranged as a polygon were 1.60 times more likely to be picked than 1943 items arranged so $(p<.001$; with 578 picking the 4 item dish and 361 picking the 3 item dish; $19595 \%$ CI 58.36\%, 64.68\%). In terms of the Plate x Items interaction, 4-items on a Square plate were 1961.24 times as likely to be chosen over 3 -items on a square plate $(p<.001$; with 540 picking the 4 197 item dish and 426 picking the 3 item dish; 95\% CI 52.70\%, 59.06\%). There was no preference 
198 when it came to the Circular plates (466 picking the 4 item dish and 466 picking the 3 item dish; 199 95\% CI 46.74\%, 53.26\%).

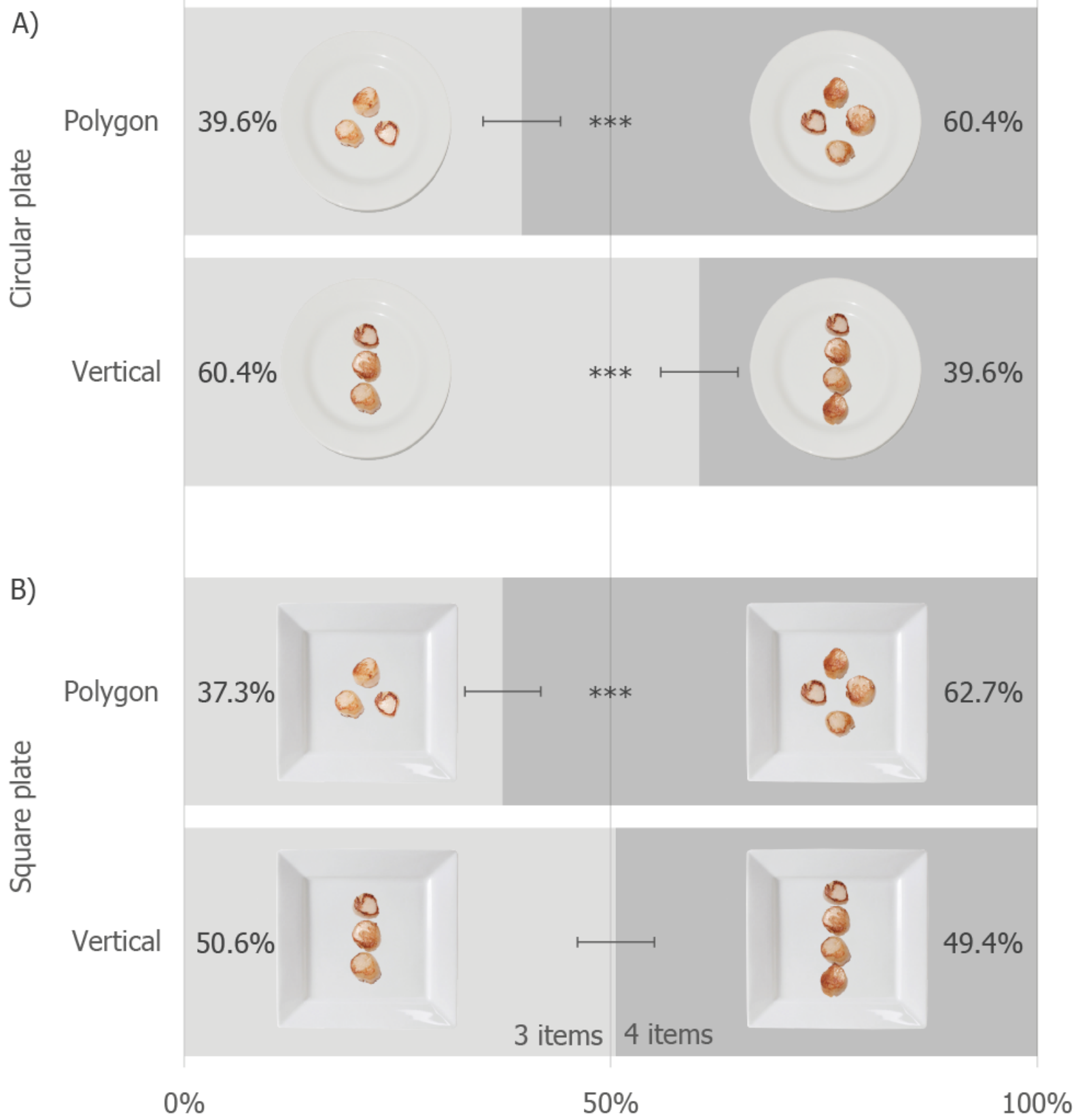


206 The findings do not generally support the hypothesis that dishes with an odd number of items

207 would be preferred over dishes with an even number of items. Although our analysis did 208 demonstrate that 3-vertically orientated scallops were preferred over 4-vertically orientated 209 scallops, visual inspection of Figure 3 shows that this effect only differed from that expected by 210 chance when the scallops were plated on a circular plate. Thus, support for the hypothesis is

211 actually more tenuous than that offered by the analysis. Indeed, overall, more evidence was found 212 for 4-items being preferred over 3-items.

213 The lack of support for the hypotheses was unexpected, and after querying social media, several 214 explanations were offered. One of the explanations proffered was that the portion sizes on 4-item 215 plates were always seen as larger than those on 3-item plates. We tested for this in Experiments $2163-6$ by varying portion size by means of scaling the images of the scallops.

217 Two other issues were also suggested via social media. The first was that the four vertical items

218 looked like substantially more food compared to those same number of items arranged as a 219 polygon, and thus the dish was not preferred over the 3-item vertical dish as there was too much 220 food on the plate. We go on to test this in Experiment 5 by asking the participants how hungry 221 they were, and testing whether this influenced the results. There was, however, no evidence for 222 such an effect.

223 The second more subtle issue was that the 4-item vertical dish looked a little less elegant to us than 224 the vertical 3-item dish, perhaps as the plate was seen as being too-full (some on social media even 225 argued that the shape of the plate was distorted, becoming more oval). To test for this, in the next 226 study, participants were exposed to dishes that were substantially larger than those used here, thus 227 preventing the dishes from seeming too full.

229 EXPERIMENT 2: Testing for a crowded plate effect

230 In this experiment we tested the hypothesis that participants found the plate crowded for the 231 vertically arranged dishes, which influenced how participants decided between a 4-item vs. a 3232 item dish. To do this, we conducted a similar study with the same factors as the previous (number 233 of items, food alignment, and plate) and included an additional factor of plate size, albeit using a 
234 repeated measures design. Specifically, besides the 'regular' sized plate used in the previous study,

235 we also collected data from those trials where a much larger plate was used instead.

236 MATERIALS AND METHODS

\section{Participants}

238

239

240

241

242

243

244

245

246

247

248

249

250

251

252

253

254

255

256

257

One hundred participants ${ }^{4}$ (35 female) were recruited from Amazon's Mechanical Turk to take part in the experiment in return for a payment of .40 US dollars. The participants ranged in age from 19 to 59 years $(M=32.0$ years, $S D=8.4)$. Only participants recorded on MTturk as originating from the United States or Canada could take part in this and all subsequent MTurk studies. The experiment was conducted on 6/06/2015, from 12:00 GMT onwards, and over a twohour period. The participants took an average of 73 seconds $(S D=52)$ to complete the study. All of the participants (here, and in subsequent studies) provided their informed consent prior to taking part in the study.

\section{Stimuli}

The 5 unique scallop stimuli used in Experiment 1 were divided into separate transparent PNG files, as were the 2 plate stimuli. The 5 scallop images were individually resized so that they all contained approximately the same number of non-transparent pixels (the original number of pixels per scallop as 41193, 44817, 42869, 33272, 42199, with a standard deviation of 4449 pixels; after resizing the stimuli so that they approximately matched the average number of pixels per scallop, the pixels per scallop were 40828, 40791, 40904, 40796, 40642, with a standard deviation on 95 pixels). The scallop image dimensions were set to 50 x 50 pixels in the actual study. The plate images were set to $250 \times 250$ pixels.

The exact scallop images used in each dish were randomly determined, as were their set positions on the plate (care was taken so that the scallops were placed and spaced apart to resemble the vertical and polygonal arrangements that had been used in Experiment 1).

\footnotetext{
${ }^{4}$ A power analysis of the ratio of 3-item to 4-item preference for circular plated vertical/polygonal food from Experiment 1 (Generic Binomial Test, using G*Power 3.1.9.2) revealed that $90 \%$ power could be achieved in this study with an $\mathrm{n}$ of 62 or 64 (the former, vertically orientated food, the latter, polygonally-orientated food). We increased this to a sample size of 100 .
} 
259 The design was similar to that of Experiment 1 in that two plates of food were shown to participants 260 on each trial, and the task was to decide on the plate that the participant most wanted to eat. Here, 261 however participants undertook all 8 of the experimental trials, which differed in terms of the size 262 of the plate shown (either both plates were large or regular sized), the shape of the plate (either 263 both were square or circular), the arrangement of the food (either both were vertical or polygonal) 264 and the number of food items (one plate there were 3-items, whilst there were 4-items on the other 265 plate).

\section{RESULTS}

267 A log-linear analysis was performed, using Plate Size (regular, large) x Plate Shape (circular, 268 square) $x$ food Arrangement (vertical, polygonal) x food Items $(3,4)$ as the variables (the final 269 model's likelihood ratio was $\left.\chi^{2}(10)=3.54, p=.99\right)$. Only the Arrangement x Items $\chi^{2}(1)=5.41$, $270 p=.021$ interaction was kept in the model. Separate Exact Binomial tests found that 4 items were 271 preferred for vertically arranged items $(p<.001$; with 307 picking the 4-item dish and 93 picking 272 the 3-item dish; 95\% CI 19.20\%, 27.70\%) and for those arranged as a polygon $(p<.001 ; 333$, $273 \quad 13.22 \%, 20.78 \%)$. 


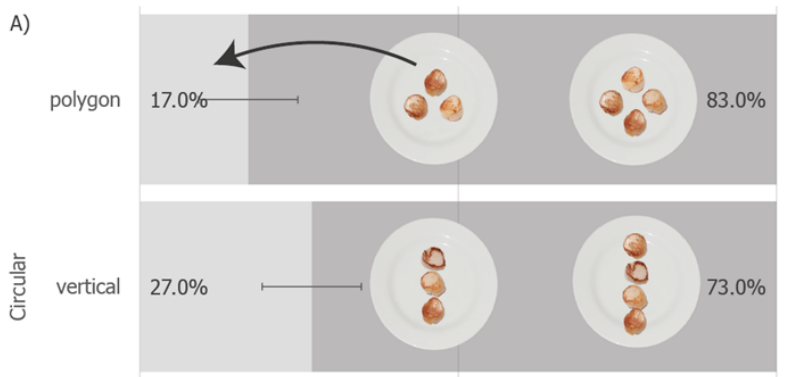

B)

\section{DISCUSSION}

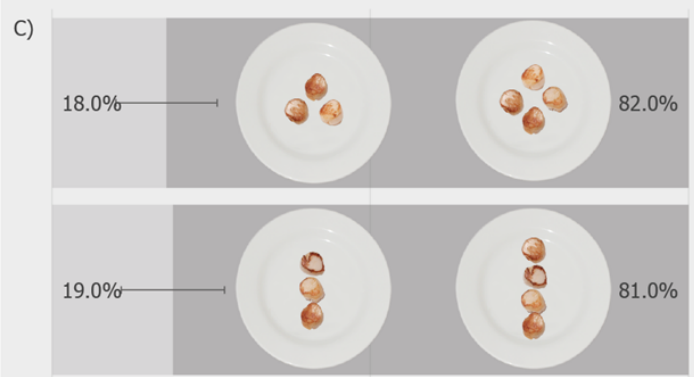

D)

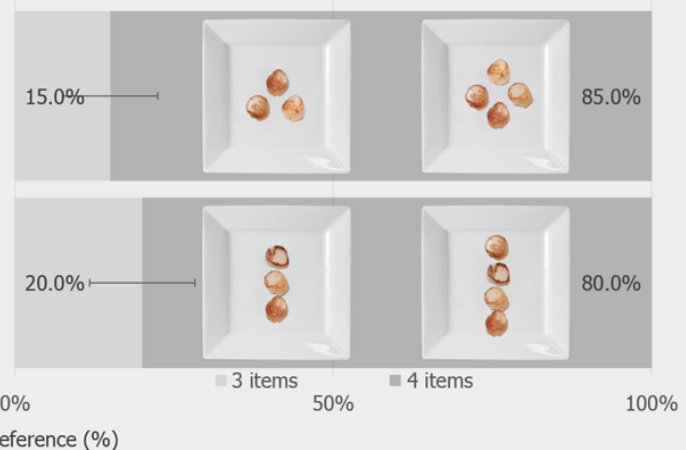

Figure 4. The percentage of participants preferring one dish over the over for each of the Plate Arrangement, Plate Size, Food Shape and Food Item cells in Experiment 2 (error bars are 95\% CI, all differences $\mathrm{p}<.001$ ).

There was no statistically significant evidence to support the scenario that plate overcrowding influenced dish selection here. At first glance, the results of Experiment 2 are rather different from those of the preceding study. Here, by far the majority of our participants preferred the 4-item dishes. In Experiment 1, though, the magnitude of this preference was much smaller; indeed, when the items were arranged vertically, participants preferred the 3-item dish over the 4-item dish. It should be noted, though, how the pattern of results in Figures 4A and 3B, which tested participants on the same plate sizes as Experiment 1, if one ignores the magnitude of the preference difference, resembles that seen in Figures 3A and 2B for Experiment 1: when the items were arranged vertically, more participants picked the 3 item dish, relative to when the items were arranged as a polygon. Given how Experiment 1 provided evidence of food arrangement and this study does not, we decided to continue exploring food arrangement in our subsequent studies. One possibility that 
290 we came up with what that we might just be looking at a ceiling effect here, and this might have

291 led to this difference between studies.

292 Why do we observe such a discrepancy between this study and the previous, in terms of magnitude 293 of preference difference? One here possibility is that the population from which the participants 294 were sampled are quite different to each other, with those in Experiment 1 predominantly coming 295 from the UK (and of the sort who visit science museums), whilst those in this study mostly came 296 from North America; indeed, a potential major driver here could be that North Americans generally

297 have larger meal sizes (as explored in the movie 'Super Size Me', Spurlock, 2004).

298

299

\section{EXPERIMENT 3: Equating portion sizes}

300 In this experiment, we scaled the 4-item dish so that it contained exactly the same amount of food 301 as the 3-item dish, by factoring in the height of the scallops. By so doing, we factor out the 302 influence of portion size in this study (if we ignore the fact that perceived portion size often differs 303 from actual portion size - as was discussed in the Introduction), which should give us a clear 304 indication whether or not participants prefer one dish over the other for perceived portion size, or 305 for the likely aesthetic difference between 3 or 4 elements being placed on the dish. Once again, 306 our hypothesis was that participants would prefer the 3-item over the 4 -item dish. Do note that 307 plate size has been shown to influence perceived portion size (for this and other such influences, 308 see Benton, 2015, and Hollands et al., 2015). However, as we never contrast portions over 309 differently-sized dishes, such effects should not confound the results reported here.

310 MATERIALS AND METHODS

$311 \quad$ Participants

312 One hundred (31 female) were recruited from Amazon's Mechanical Turk to take part in the 313 experiment in return for a payment of .35 US dollars. The participants ranged in age from 18 to 69 314 years $(M=33.1$ years, $S D=10.9)$. The experiment was conducted on 10/06/2015, from 16:00 315 GMT onwards, and over a one-hour period. The participants took an average of 89 seconds $(S D=$ 316 104) to complete the study. 


\section{Stimuli, Design and Procedure}

318 This study was identical to Experiment 2 except that the scallops were scaled so that each plate

319 contained the same amount of food. In the previous studies, the scallops were held within 50 x 50

320 pixel boxes, and we assumed that the height that the scallops were off the plate was approximately

$3212 / 3$ of this measure (33.3 pixels). Thus, on a three-item plate, the scallops were each tightly held

322 within a 250000 voxel box $(3 * 50 * 50 * 33.33)$. The scallops in the four-item plate were scaled

323 along the $\mathrm{x}, \mathrm{y}$, and $\mathrm{z}$ axes to $90.86 \%$ of their original size so that the boxes they were enclosed

324 within also summed up to this value $(4 \times 45.43 \times 45.43 \times 30.29)$.

325

326

327

328

329

330

331

332

333

334

335

336

337

338

339

340

$341 \quad$ Participants

\section{RESULTS AND DISCUSSION}

A log-linear analysis, as defined in Experiment 2, was conducted using data from this study (the final model's likelihood ratio was $\left.\chi^{2}(14)=5.23, p=.98\right)$. As in the previous study, the model only retained the effect of Items $\chi^{2}(1)=41.77, p<.001$. 4-item dishes (selected 491 times, or $61.38 \%$ of the time) were 1.59 times more likely to be preferred more than dishes with 3 items (309 times; Fisher's exact t-test 95\% CI were $57.90 \%$ and $64.76 \%$ ).

The results indicate that, in actual fact, the 4-item dishes were preferred over the 3-item dishes. This result certainly runs contrary to the widespread claim that that odd-number of items are preferable. Unfortunately, however, a further confound may have swayed this result: Could portion sizes have been distorted by some other means? Next, we tested whether our arrangements were thought different in portion size due to potential distortions brought about by psychological illusions of volume perception.

\section{EXPERIMENT 4: Scaling study}

MATERIALS AND METHODS 
342 One hundred participants (51 female) were recruited from Amazon's Mechanical Turk to take part

343 in this study in return for a payment of 1 US dollar. The participants ranged in age from 19 to 56

344 years $(M=30.2$ years, $S D=8.02)$. The experiment was conducted on 5/06/2014, from 14:00 GMT

345 onwards, over a period of three-hours. The participants took an average of 378 seconds $(S D=138)$

346 to complete the study.

\section{Stimuli}

348 The individual scallops used in Experiment 2 onwards were dynamically sized, positioned and combined as a dish stimulus as required on each trial (on a plate in most trials; n.b., the plates used were those defined in Experiment 2). The exact scallop images used in each dish that were to be scaled (henceforth termed the 'scaling-dish') were selected randomly, as were their set positions on the plate (care was taken so that the scallops were placed and spaced apart to resemble the vertical and polygonal arrangements that had been used in Experiment 1). The scallops in each dish were simultaneously scaled using the scroll button on the mouse or the left and right cursor

355

356

357

358

359

360

361

362 keys (where a 'toward the body' scroll and the left cursor key scaled the image downwards) importantly, the distance between the centre points of the targets did not change on scaling. The minimum size scallops could be scaled so that they tightly fit within a $25 \times 25$ pixel box. The maximum size was $150 \times 150$ pixels. The starting size of the scallops was randomly determined but was always such that the scallops fit within a box larger or equal to $40 \mathrm{x} 40$ pixels and smaller or equal to $60 \times 60$ pixels.

A target stimulus that was randomly selected from the 5 scallop stimuli was also present on each trial. This stimulus was always sized so that it fit within an 87 x 87 pixel box.

\section{Apparatus}

The apparatus varied by participant as the experiment was conducted online. The experiment utilized 'full screen' mode (i.e., utilizing the entirety of the participant's monitor), and took place within a 1024x768-pixel box in the centre of the screen, irrespective of the size of the monitor. The experiment was conducted on the Internet using the Adobe Flash based version of Xperiment (http://www.xperiment.mobi). 


\section{Design}

371 A within-participants experimental design was used with all of the participants undertaking all of

372 the experimental trials (trial order was randomised). The dependent variable was the computed 373 scaling factor which the participant applied to the dish of scallops so that they would, together, 374 match the volume of the Target stimulus. A scaling factor of 1 would indicate that the participants 375 scaled the portion so it exactly matched the volume of the target, whilst values smaller than 1 376 indicate the scallops were sized such that they were of a lesser overall volume than the target. 377 Independent variables were the size of the plate (large or regular), the shape of the plate (circular 378 or rectangular), the number of scallops (three or four) and the arrangement of the scallops (vertical 379 or polygonal). Further trials included dish variants where there were 1 or two scallops only (the 380 latter, arranged vertically or horizontally) and where there was no plate present. Note that there 381 were several further conditions, the data from which will be reported elsewhere (Woods et al, in 382 prep.).

\section{Procedure}

385 On each trial, a screen as shown in Figure 5 was presented. Participants had to scale the size of the 386 scallops shown in the portion of the screen labelled Portion 2, so that they matched the same amount of food as shown in Portion 1. Although Portion 1 was the same size on all trials, the Scallop that was shown as Portion 2 randomly varied in default size across trials. There were a total of 35 trials. At the end of the study we explicitly asked participants "When you did the task, were you resizing Portion 2 so that...", and offered two choice options "one food item was the same size as Portion 1", "all the items together in Portion 2 added up to the same amount as in

392 Portion 1." The 20 participants who chose the first option were excluded from the analysis. There 393 were 35 experimental trials, the data from 20 being reported here. 
Please use the scroll button on your mouse, or your left/right keys, to resize all the items in portion 2 so that they are the same amount of food as portion 1.
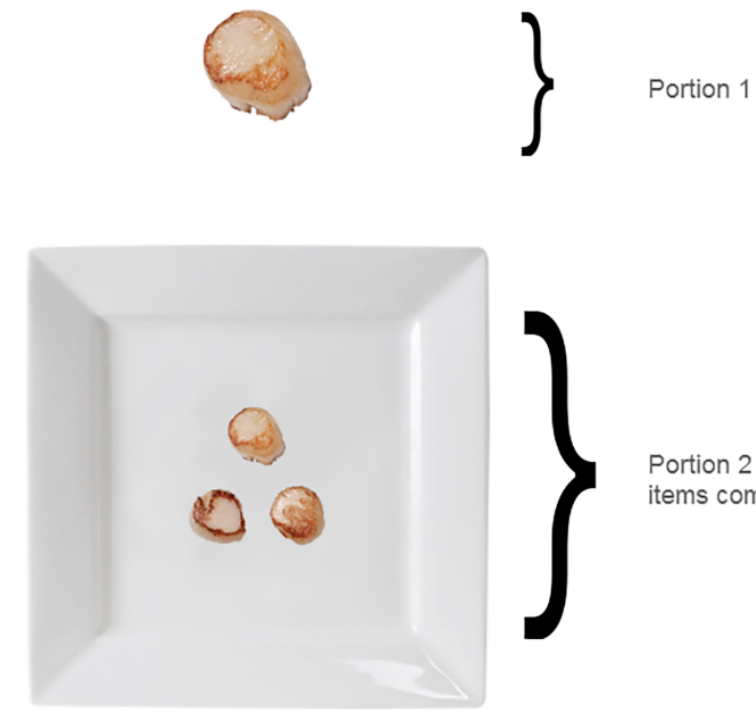

Portion 2 (all the items combined)

next (SPACE)

Figure 5. The trial layout, as presented to the participants in Experiment 4.

\section{RESULTS AND DISCUSSION}

399

400

401

402

403

404

405

406

407

408

409

Eleven out of twenty sets comprising the data were not normally distributed $D(80), p<.05$. $\log$ transforming the data mostly corrected this issue, with only one set remaining non-normal, $D(80)=.94, p<.001$ (large round plates containing 3 polygonally-arranged items). The same set was also was significantly skewed, $p<.001$, and affected by kurtosis, $p<.01$. Another set was also affected by kurtosis, $p<.01$ (regular-sized round plates with 4 vertical items). $0.5 \%$ of the scaling data from each dish was found to be outlying (defined as being larger or smaller than the mean +-3 standard deviations) and so was corrected (replaced with the nearest non-outlying data value, mean +-3 standard deviations).

With the majority of the cells of data now being normally distributed, one-sample t-tests were used to test whether the log-scores different from the null hypothesis of that no scaling was required, or $\log (1)$, with a Bonferroni corrected alpha threshold set to $.05 / 35$ (a further 15 tests on data not 
410 reported here were conducted in Woods et al., in prep.). Only data for large round plates with 4 411 vertical scallops differed significantly $t(79)=3.64, p<.001$, requiring scaling of 1.10 to be seen as 412 the same size as the target food. As all other 4-scallop dishes did not so differ (as would be expected 413 given the shift in 3 vs. 4 item preference seen in previous studies), we must assume the null414 hypothesis that portion size distortions cannot really account for earlier findings (that 4-item 415 portions were often preferred over 3-item portions).

416 Note, though, that in previous research the participants had to choose between 2 dishes, each of 417 which could be differently influenced by scaling factors. Thus, potentially subtler distortions of 418 size (not detectable when contrasting from baseline as done in the above tests that were essentially 419 between-participant), between each pair of dishes, may have driven the shift towards the 4-item 420 dish as opposed to 3-item dish from past studies. To explore this, a 4-way repeated measures 421 ANOVA was conducted on the log scaling data with plate Shape, plate Size, Items and food 422 Arrangement as factors. Items and Arrangement interacted $F(1,79)=22.86, p<.001, \eta_{p}{ }^{2}=.22$, with 423 a post-hoc stepwise Newman-Keuls analysis showing that 4-scallops arranged as a polygon 424 requiring more scaling (mean 1.04) than the other conditions (.97; significant main effects that 425 were involved in these interactions are not reported). What this means, in fact, is that the 4-scallop 426 polygon arrangements required were seen as the smaller portion than on other dishes (it was 427 required to be scaled by a factor of 1.04 , whilst the other dishes had to be scaled by .97 , to both be 428 seen as the same size as the target portion). We would have expected it to be seen as bigger than 429 the other dishes, for it to explain the apparent 4-item preference over 3-items as seen previously.

430 Recall the pattern of results from Experiments 1 and 2, where the preference ratio of 3-item 431 polygonal scallops to 4-item polygonal scallops was greater or more severe than that for vertically 432 arranged items. The fact that here, 4-items are perceived as a smaller portion than 3-items may be 433 linked to this pattern, although at this moment in time, it is unclear how.

434 Several other distortions, albeit smaller in magnitude, were also found. Shape and Size also 435 interacted $F(1,79)=5.85, p<.018, \eta_{p}{ }^{2}=.07$, with the same post-hoc procedure revealing that large436 round plates required its contents to be scaled more to match the target (mean 1.02) as compared 437 to regular-round (.97), large-square (.99) and regular-square plates (.98). Large-square plates 438 required more scaling than round-regularly sized plates. 
439 A separate repeated measures ANOVA was used to test whether the trials in which there were no 440 plates (only scallops were shown) differed in terms of scaling required to match the target stimulus. 441 Items and Arrangement were used as factors. There was a main effect of Items, $F(1,79)=8.47$, $442 p=.01, \eta_{p}{ }^{2}=.10$, with the 4 -item displays (mean 1.04) requiring significantly more scaling than the 443 3-item displays (1.00).

444

445

\section{DISCUSSION}

446 Although some stimuli from previous experiments were indeed affected by size distortions, there 447 was no systematic effect of distortion of 4-item dishes to appear larger than the 3-item dishes, the 448 result of which could be leading participants to prefer 4-items over 3.

449 The tentative conclusion that could now be drawn is that the even number of items on a plate are 450 preferred over odd numbers of items. To say so, though, one must ignore several important issues 451 highlighted in the introduction, such as whether 3 vs. 4 items generalise to odd vs. even number 452 of items, as well as whether the effects observed here are only applicable to our scallop stimuli.

454 COMBINED ANALYSES

455 The preceding experiments have highlighted the importance of perceived portion size on dish 456 choice, with larger portions tending to be selected over smaller portions. This relationship has been 457 quantified in Figure 6 as a simple-regression model, which shows an extraordinary linear 458 relationship between these factors, for all studies, except the very first one. 


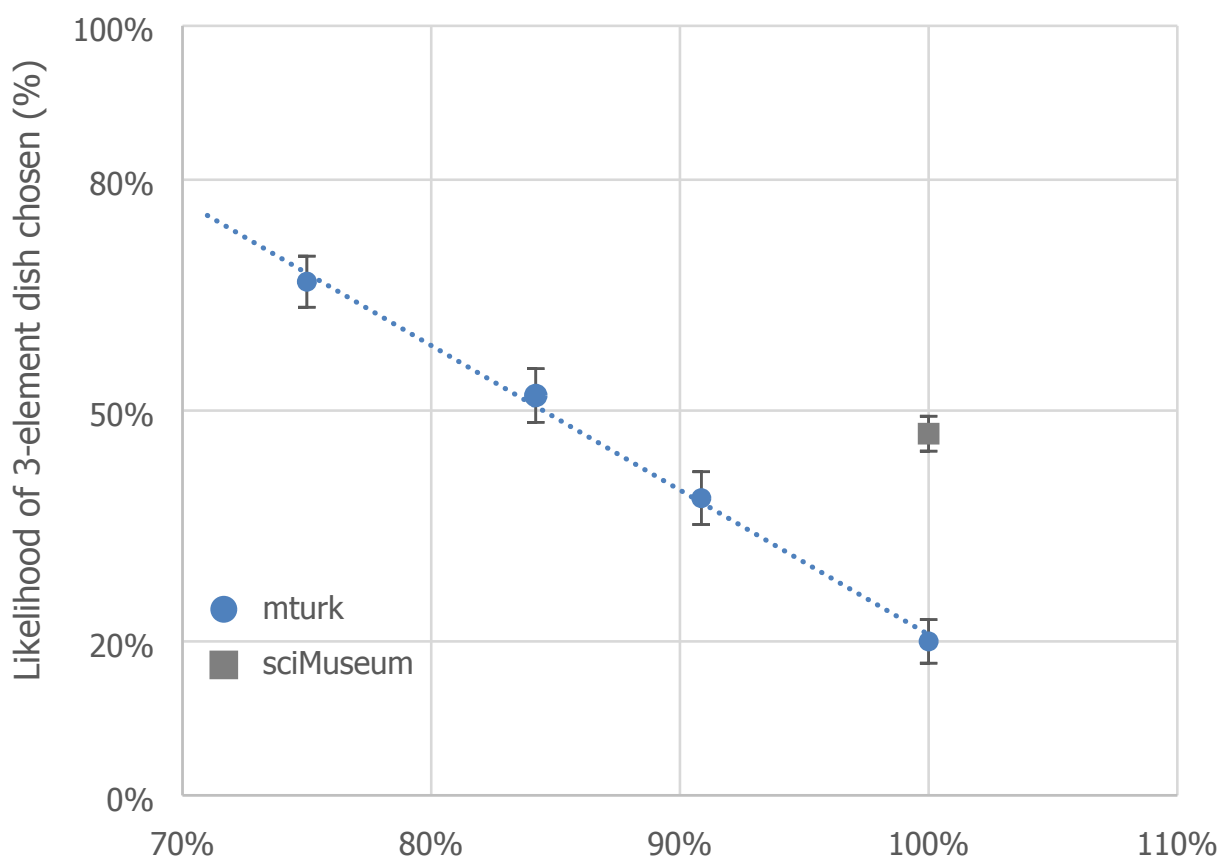

Scaling of individual 4-item scallops relative to 3-item scallops

460

461 Figure 6. Depiction of the relationship between 4-item scallop scaling and likelihood of 3-scallop dish 462 chosen, over the experiments reported so far. Error bars represent the 95\% CI derived from separate Fisher's 463 exact binomial tests.

465 So, the question remains as to why the results from the Science Museum study differ so much from 466 the data collected from Mechanical Turk for Experiments 2-5? Recall that the scallops in our 467 original study were not yet scaled to be equal in size in terms of pixels, as done from Experiment 4682 onwards. Could the 'fixed' stimuli used in Experiment 1 have led to the above discrepancy? To 469 test for this, we isolated each dish in the study using photo-editing software to estimate total scallop 470 pixels (see Table 1). We then calculated the individual scaling factor present for each condition 471 (square plate $\mathrm{x}$ vertical items, .98; square x polygon 1.03; circular x vertical, .93; circular x 472 polygon, 1.04) and plotted this on Figure 7, alongside the ratio of 3-items being selected for each 473 condition.

474 Table 1: Detailing the size, in pixels, of each scallop that was used in Experiment 1.

\begin{tabular}{|c|c|}
\hline Plate shape & Food shape \\
\hline
\end{tabular}




\begin{tabular}{llccc}
\hline Circle & polygon & 3 & 4606 & 1711 \\
Circle & polygon & 4 & 6612 & 1702 \\
Circle & vertical & 3 & 5107 & 1650 \\
Circle & vertical & 4 & 5919 & 1653 \\
Square & polygon & 3 & 4545 & 1535 \\
Square & polygon & 4 & 6427 & 1607 \\
Square & vertical & 3 & 5133 & 1480 \\
Square & vertical & 4 & 6598 & 1515 \\
\hline
\end{tabular}

475

476

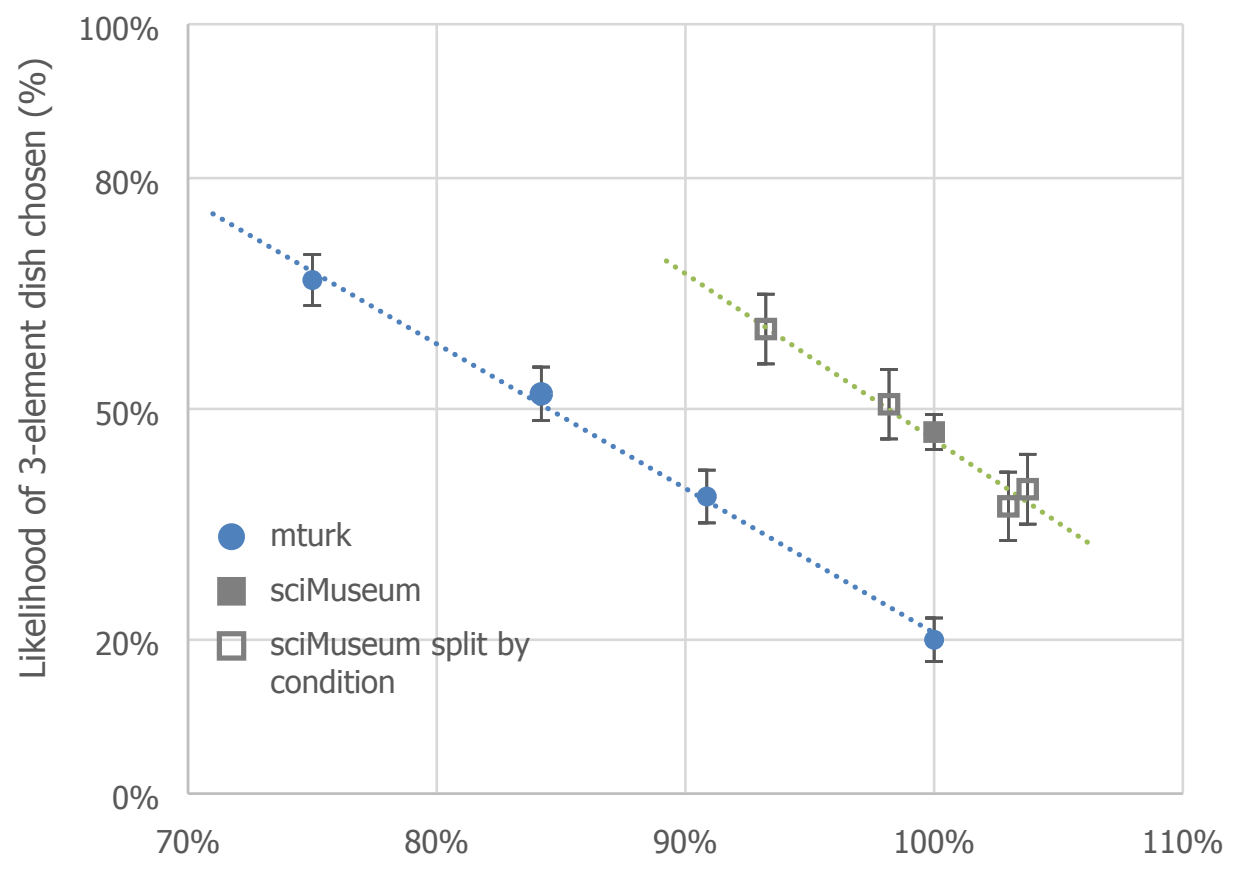

477

Scaling of individual 4-item scallops relative to 3-item scallops

478 Figure 7. Identical to Figure 6, except that the conditions from Experiment 1 have been added individually as 479 transparent black bordered squares. 
481 Although with 4 data points per model, any inference from statistical analysis must be treated with 482 considerable caution, the updated MTurk model's gradient (-.53, 95\% CI -.63, -.43) and Science 483 Museum model's gradient (-.45, -.67, -.24) are similar; it is their y-axis intercepts that potentially 484 differ (Experiment 2-5, 111\%, 106\%, 116\%; Science Museum, 121\%, 110\%, 131\%; n.b. 485 overlapping CI).

486 Why would there be this upward shift of preferring 3-items as opposed to 4 in the Science Museum 487 study? After further careful investigation we discovered that the images that had been used in the Science Museum study had themselves been arbitrarily scaled by the designers of the citizen science platform that they had been presented on so that they were $67.1 \%$ smaller in width and height than their original file size (images contained within a 380 pixel x 255 pixel image-file).

491 Furthermore, it also transpired that there were two sizes of the original stimuli, with the original

492 images we used being both smaller in scale and held within a differently dimensioned image-file 493 (372 pixels x 306 pixels). By using graphical editing software, we were able to estimate that the 494 Science Museum images were 85.2\% smaller in width and height to the images used in 495 Experiments 2-5. Could the difference in overall food size lead to this apparent upward shift between models as seen in Figure 7? We test this hypothesis next. We also tested whether participants' hunger influenced their dish choice.

\section{EXPERIMENT 5: Difference due to overall size of stimuli?}

500 In this study, participants undertook a version of the task reported previously where we 501 systematically varied the physical sizes of the dish stimuli on the screen. Both 3-and 4-portion 502 stimuli were resized to the same degree. Note that the monitors of our online participants and thus 503 the stimuli presented differ in terms of size across individuals. To get around this issue, we used a 504 repeated measures design such that all of the participants undertook the trials where differently 505 sized stimuli were presented.

506 It was hypothesised that if the variation in the size of the stimuli was indeed responsible for the 507 difference between the Science Museum study and all of the other studies (as shown in Figure 7), 
508 in this study, we should observe a shift in dish preference as we scale the stimuli from smaller to

509 larger in size from that observed for the Science Museum study to that observed for the Mechanical

510 Turk experiments.

511 We also tested whether the participant's self-reported hunger level influenced the choice design in

512 this task by asking participants how hungry they were.

\section{MATERIALS AND METHODS}

514 One hundred participants (40 female) were recruited from Amazon's Mechanical Turk to take part

515 in the experiment in return for a payment of .50 US dollars. The participants ranged in age from

51620 to 67 years $(M=34.8$ years, $S D=11.2)$. The experiment was conducted on $15 / 06 / 2015$, from

517 14:30 GMT onwards, and over a 45-minute period. The participants took an average of 105

518 seconds $(S D=58)$ to complete the study.

520 The stimuli were the same as reported in Experiment 1, except that the scaling of both the 3-item 521 and 4-item dishes (as well as plates) were varied, relative to the original size of the 3-item stimuli

522 as used in Experiment 2. We decided to size the stimuli at 100\% of those used in Experiment 2 (50 523 pixels along one dimension), same size of the Science Museum study (42.6 pixels; a difference of 5247.39 pixels), smaller than the Science Museum by 7.39 pixels, and larger than the one used in 525 Experiment 2 by 7.39 pixels. In order of size, the stimuli were scaled to $70.44 \%, 85.22 \%, 100 \%$ 526 and $134.28 \%$ of the stimuli used in Experiment 2 and onwards (henceforth termed Small, 527 SciMuseum, Regular, Large).

\section{Design}

529 We used a fully factorial design here with all participants completing all of the experimental trials. 530 The design was identical to that in Experiment 1, except that an additional factor of plate Size 531 (regular versus large) was included. We also had the participants report their hunger level.

\section{$532 \quad$ Procedure}


533 The procedure was identical to that used in the previous studies except that we also assessed 534 participants' self-reported hunger by means of scaled anchored on the left hand side with "not 535 hungry at all" and on the right "very hungry". Hunger scores from this scale varied from 0 to 100.

537 A log-linear analysis was performed, as defined in Experiment 2, but with the additional 538 independent variable of plate Size, using the data from this study (the final model's likelihood ratio was $\chi^{2}(30)=5.12, p=1$. The only factor to be retained by the model was Items, $\chi^{2}(1)=138.91$, $p<.001$, with 4-item dishes (selected 1034 times) 1.83 times more likely to be chosen than 3 -item dishes (selected 566 times). The Exact Binomial test 95\% confidence intervals for this effect $(33.03 \%, 37.78 \%)$ intersected the value predicted by the model for a scaling of $90.86 \%$ for the 4 item scallops (37.62\%). The lack of any effect of Size means that the Small (3-items chosen $54433.50 \%$ of the time, $95 \%$ CI $28.89 \%$, 38.36\%), SciMuseum (35.00\%, 30.33\%, 39.90\%), Regular $(36.75 \%, 32.01 \%, 41.68 \%)$ and Large sizes $(36.25 \%, 31.53 \%, 41.17 \%)$ did not significantly differ from each other in terms of the ratio of participants who chose 3-item versus 4-item dishes.

547 To test whether the hunger level of the participant influenced their dish choice, a correlation 548 analysis was used to test for a relationship between the total number of times each participant chose the 4-item dish, and their self-reported hunger score. As the 4-item dish was 1.83 times more likely to be chosen than the 3-item dish (as reported above), we would then expect that, if hunger was an important factor, participants who were more hungry would be more likely to choose the 4-item larger in portion size dish, $r=-.12, n=100, p=.25$.

There was no evidence that the difference in size between stimuli used in Experiments 2-5 and those used in Experiment 1 was responsible for the difference in y-axis intercept. There are several possible reasons for this. One possibility is that the within-participants design of this study could have prevented any effects being detectable. For example, consider that the participants here saw many trials one after the other, involving the same task, "Which dish do you prefer"? Potentially, after undergoing several such trials, the participants may have 'made up their mind' as to how to respond to each trial (e.g., "I like big portions, so I will always pick the larger portion"), which could sufficiently dilute any normally detectable effects so that they became undetectable. In the Science Museum task, however, the maximum number of trials undertaken by the participants 
562 were 2, with the majority of trials thus requiring cognitive effort rather than relying on a quick 563 heuristic.

564 Another possibility is that the populations from which participants from Experiments 2-6 were

565

566

567

568

569

570

571

572

573

574

575

576

577

578

579

580

581

582

583

584

585

586

587

588

\section{EXPERIMENT 6: Do the effects hold when sampling from a different population?}

A logical step is to rerun the study, but with a different group of participants. Psychology students are well known for being WEIRD (Western, Educated, Industrialised, Rich, and Democratic individuals; see Henrich et al., 2010) and different from Mechanical Turkers (discussed in Woods et al., 2015). Here, we recruited participants from the up-and-coming cloud-sourcing platform Prolific Academic, which actively recruits participants with no geographic criteria for potential participants (although if desired, am extensive range of filters can be used to define the subpopulation of participants one needs for a given study), as opposed to MTurk, whose participants are typically North American.

If the difference between the data from the Science Museum reported in Experiment 1, and the rest of the studies reported so far is indeed attributable to some difference over populations, Prolific Academic participants may differ from both these groups too.

To test whether this is so, we collected data from stimuli that are sized according to those reported in Experiment 2, 3, and Supplementary Experiments A, and B. We should observe the same gradient as found previously, but with a shift in the y-axis intercept.

\section{MATERIALS AND METHODS}

\section{Participants}

391 participants (162 female) were recruited from Prolific Academic to take part in this study in return for a payment of .35 US dollars. The participants ranged in age from 18 to 67 years $(M=$ 28.4 years, $S D=9.1)$. 142 participants reported being from the United States, 135 from the United Kingdom, 17 from India, 13 from Canada and 5 from Portugal (country frequencies of fewer than 
5895 individuals are not reported). The experiment was conducted on 3/07/2015, from 16:00 GMT

590 onwards, over a period of six-hours. The participants took an average of 106 seconds $(S D=48)$ to

591 complete the study.

592

Stimuli, Design, Procedure

593 Identical to Experiment 2, except that Large Plate condition was removed and an additional

594 between participant factor of Scaling was included (how large the 4-item stimuli were, relative to

595 the 3-item stimuli, the levels being $100 \%, 91 \%, 84 \%$, and $75 \%$ ).

596 RESULTS and DISCUSSION

597 A log-linear analysis was run using data from this study (the final model's likelihood ratio was $598 \chi^{2}(14)=3.80, p=1$. Items $\times$ Scaled $\mathrm{x}$ Arrangement interacted $\chi^{2}(3)=10.54, p<.02$. Eight separate 599 Bonferroni corrected Fisher's Exact tests were used to explore this interaction, the results of which 600 are detailed in Table 2.

601 Table 2. The percentage preference for 3-items relative to 4-items in Experiment $6(* * \mathrm{p}<.01, * * * \mathrm{p}<.001$, as derived 602 from Bonferroni-corrected exact Fisher's tests; 95\% CI in brackets).

\begin{tabular}{rrrrr} 
& \multicolumn{4}{c}{ Scaling of 4-item scallops to 3-item scallops } \\
\cline { 2 - 5 } & $100 \%$ & & & \\
\cline { 2 - 5 } Polygonal & $30.39 * * *$ & $25.27 * * *$ & 44.33 & $65 \%$ \\
arrangement & $(24.16,37.20)$ & $(19.20,32.15)$ & $(37.22,51.62)$ & $(55.06,69.06)$ \\
Vertical & 47.55 & 45.70 & 49.48 & $64.29 * * *$ \\
arrangement & $(40.53,54.64)$ & $(38.39,53.15)$ & $(42.25,56.74)$ & $(57.15,70.99)$ \\
$\mathrm{n}$ & & & & 194 \\
\end{tabular}

603

604 The Items x PlateShape interaction was also significant, $\chi^{2}(1)=4.34, p<.05$ with follow-up Exact 605 Fisher tests for each Plate Shape revealing that Square Plates with 4 items (selected 440 times) 606 were selected 1.29 times more frequently than Square Plates with 3-items $\mathrm{p}<.001$ (selected 340 607 times). There was no such difference for Round Plates (3-items selected 381 times, and 4-items 608399 times).

609 The data for this experiment has been plotted alongside the previous experiments in Figure 8.

610 Whilst the scaled data points for $91 \%, 84 \%$ and $75 \%$ form a straight line that does not appear to 
611 differ from that of the past MTurk experiments (gradient, -.57, 95\% CI -.64, -.5; intercept 111\%, $61295 \%$ CI, $107.53 \%, 114.56 \%$ ), the data from the $100 \%$ scaled condition unexpectedly does not fit 613 this profile (the transparent purple point in the Figure).

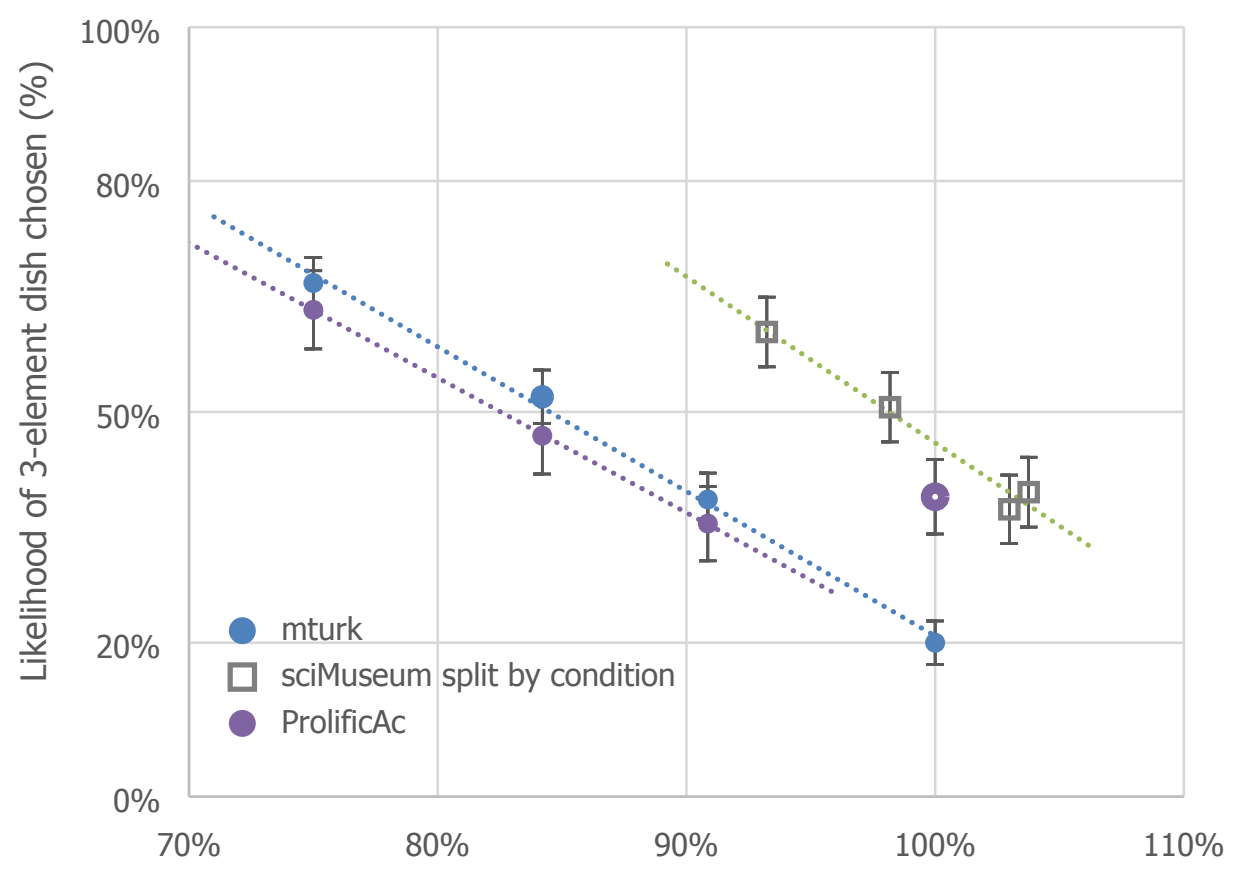

Scaling of individual 4-item scallops relative to 3-item scallops

614

615 Figure 8. Identical to Figure 7 but with the results of Experiment 6 added. Note that the large transparent purple point 616 did not follow the pattern of the other data points from this study.

617

618 Back in Experiment 2 (labelled E2 in Figure 8, in the lower right quadrant along the X-axis 100\% 619 mark) we observed an Arrangement $x$ Items interaction and speculated that this arose due to 620 overcrowding on the plate for the 4-item in relation to the 3 -item vertical dishes. One possibility 621 in the current study is that the vertically aligned scallops were likewise seen as overcrowding the 622 plate. For some reason, however, the participants here preferred this compared to when the items 623 were not so overcrowded, hence the 4-item preference from 3-item preference shifted upward, as 624 shown in the graph.

625 Unfortunately, due to the confound of stimuli sizing for the Science Museum study discussed in 626 the COMBINED ANALYSES section, we do not have data for Vertical dishes at this level of 4-item 
627 scaling. We do, however, speculate that such an effect would be present, and would increasingly

628 influence the results as overcrowding increased yet further. As overcrowding is not, however, the

629 focus of the present research, we will leave the speculations of the drivers of this finding to future 630 research.

631 In terms of our initial hypothesis, despite the above unexpected finding, there is little evidence to 632 support the idea differences in terms of population led to the shift in intercept between MTurk 633 studies reported here, and the results of the Science Museum. In the General Discussion, we flesh 634 out reasons why this may be the case.

635

\section{EXPERIMENT 7: 1-6 scallops per plate}

637 Our reviewers rightly pointed out a potential additional confound that we had missed, that of 638 'numerosity'. In all of the past studies that have been reported so far, participants were shown 639 dishes where the one with an odd number of items always contained fewer items than the dish 640 containing an even number of items. It could therefore be, that rather than participants referring an 641 even number of items, that they simply preferred the plate with more food items on it, as has been 642 previously observed in both animals and human infants (see Hauser, Carey \& Hauser, 2000; Uller 643 \& Lewis, 2009).

644 Here, we test a range of plate pairs containing one to six elements, with some plate pairs (all of 645 which whose food was scaled, as done previously, to appear to contain the same amount of food) 646 where the odd numbered plate has fewer elements than the even numbered plate, and where the 647 even numbered plate has more than the odd number. We also test pairs of plates that both contain 648 differing numbers of odd items, and even items. No variation in choice would be expected over 649 these plate-pair configurations if it is numerosity that drives the previously observed effects.

\section{MATERIALS AND METHODS}

651 One hundred participants (44 female) were recruited through Prolific Academic to take part in the 652 experiment in return for a payment of .45 UK pounds. The participants ranged in reported age 653 from 18 to 60 years $(M=33.7$ years, $S D=11.9)$. The experiment was conducted on $8 / 11 / 2015$, 
654 from 10:00 GMT onwards, and over a 3-hour period. The participants took an average of 169 655 seconds $(S D=61)$ to complete the study.

656 Stimuli, Apparatus

657 The scallop images were the same as reported in the previous study. These were combined into 658 stimuli of 1-6 scallops, with the individual scallop-images scaled so that the stimuli contained the 659 same amount of food (using the procedure incorporating depth, as detailed in Experiment 3; the 660 scaling factor used for $\mathrm{x}, \mathrm{y}, \mathrm{z}$ dimensions of 1, 2, 3, 4, 5, 6 scallop stimuli were respectively 1.44, $6611.14,1,0.91,0.84,0.79)$. The initial scallop-image was placed centrally. Subsequent image 662 positions were determined by randomly selecting one of the already placed images and then 663 generating a random point 55 to 82.5 pixels from its centre. If the new position was within 55 664 pixels of any already placed image, the process was repeated. If this was unsuccessful after 10,000 665 attempts (this never occurred during development, with typically $<100$ attempts needed per 666 placement), the point of farthest distance from existing images so far generated was used. This led 667 to stimuli consisting of scallop-images that were clustered around a central point. When needed, a 668 sixth scallop-image was chosen at random from the 5 original scallop images. The pair of stimuli 669 shown on each trial differed in terms of the number of scallop-images, with this number always 670 being by 1 or scallops (the pairs consisted of: 6 vs 5 scallops, 6 vs 4, 5 vs 4, 5 vs 3, 4 vs 3, 4 vs 2 , 6713 vs 2,3 vs 1,2 vs 1$)$.

\section{Design}

673 We used near fully factorial design here with all participants completing all of the experimental 674 trials. The independent variables were the number of scallops on each plate (ranging from 1-6) and 675 the difference in this number of scallops over plates in a stimulus pair (1 or 2; n.b. we could not 676 test 2- vs 0 -items, thus our design is incomplete). The dependent variables were the dish out of 677 each pair that was preferred, and, as a control, the dish that the participants thought contained the 678 most food.

\section{Procedure}

680 The procedure was identical to that used in the previous study. As before, participants had to select 681 which of two stimuli they preferred (there were 9 such trials). After, the participants were shown 
682 the same pairs of stimuli (that were identical in terms of individual scallop position) and ask for 683 each, which dish contained the most food. There were 18 trials in total.

684

685 RESULTS and DISCUSSION

686 To test whether the groups differenced in terms of whether the dish with the most items was 687 selected in preference over the dish with a fewer number of items, all 9 groups were entered into 688 a log-linear analysis under the factor of Group, along with this Most Preferred factor (see Table 689 3). The model, whose likelihood ratio was $\chi^{2}(16)=14.71, p=.55$, only retained the Most Preferred factor $\chi^{2}(1)=53.33, p=.001$, with participants 1.63 times as likely to choose the dish that contained

691 more scallops as opposed to the plate with fewer scallops. The analysis was repeated with 692 dependent variable of Most Amount (whether the participant had chosen the dish with the most 693 items as containing more food, over the dish with the fewer items) instead of the Most Preferred 694 factor (likelihood ratio was $\chi^{2}(16)=11.52, p=.77$ ). The factor of Most Amount was (barely) retained $695 \chi^{2}(1)=4.00, p=.04$, with dishes with fewer items thought as containing more food 1.14 times than 696 dishes with more items. The result could indicate that participants preferred the dishes with 697 seemingly less food on them. If this were so, we should expect that the difference in the perceived 698 amount of food to tally with the degree to which a plates differed in preference; with our limited 699 sample, we conclude, be it very tentatively, that there was no evidence for this, $r=.27, n=9, p=.48$. 700 Another consideration is that hungry people typically prefer larger portion sizers (e.g. Brunstrom, 701 Rogers, Pothos, Calitri \& Tapper, 2008), so if our effect of smaller portions being seen as larger 702 did hold sway in people's dish preference, one would expect hungrier individuals to be more so 703 influenced. However, when we re-ran the original log-linear analysis but including an additional variable of median split (which was 52.02 on our hunger scale; $1^{\text {st }}$ and $3^{\text {rd }}$ quartiles were 23.29 and 72.12), this factor was not included in the final model. ${ }^{5}$

Table 3. The percentage preference for the different dish pairs in Experiment 7, sorted according to preference magnitude $(* \mathrm{p}<.05, * * \mathrm{p}<.01, * * * \mathrm{p}<.001$, as derived from Bonferroni-corrected exact Fisher's tests; 95\% CI in brackets). Note how the column defining whether the dishes were odd or even seems relatively random in terms of order, which implies no relationship between this and strength of Preference.

\footnotetext{
${ }^{5}$ The model, whose likelihood ratio was $\chi^{2}(34)=22.33, p=.94$, again only retained the Most Preferred factor $\chi^{2}(1)=53.33, p=.001$.
} 


\begin{tabular}{|c|c|c|c|c|c|}
\hline \multicolumn{3}{|c|}{ Items } & \multirow[b]{2}{*}{$\begin{array}{l}\text { Dish1, Dish2, odd } \\
\text { or even items }\end{array}$} & \multicolumn{2}{|c|}{ Dish with more elements, by $\%$ of participants, is } \\
\hline Dish1 & Dish2 & Difference & & Preferred $(95 \% \mathrm{CI})$ & $\begin{array}{l}\text { Bigger portion } \\
\quad(95 \% \mathrm{CI})\end{array}$ \\
\hline 1 & 3 & 2 & $\mathrm{OO}$ & $73(63.2,81.39) * * *$ & $47(36.94,57.24)$ \\
\hline 2 & 4 & 2 & $\mathrm{EE}$ & $70(60.02,78.76) * *$ & $40(30.33,50.28)$ \\
\hline 4 & 5 & 1 & $\mathrm{EO}$ & $66(55.85,75.18) *$ & $42(47.71,67.8)$ \\
\hline 2 & 3 & 1 & $\mathrm{EO}$ & $62(51.75,71.52)$ & $42(32.2,52.29)$ \\
\hline 1 & 2 & 1 & $\mathrm{OE}$ & $62(51.75,71.52)$ & $44(34.08,54.28)$ \\
\hline 3 & 5 & 1 & $\mathrm{OO}$ & $59(48.71,68.74)$ & $50(39.83,60.17)$ \\
\hline 5 & 6 & 2 & $\mathrm{OE}$ & $57(46.71,66.86)$ & $48(37.9,58.22)$ \\
\hline 4 & 6 & 2 & $\mathrm{EE}$ & $57(46.71,66.86)$ & $60(30.33,50.28)$ \\
\hline 3 & 4 & 1 & $\mathrm{OE}$ & $53(42.76,63.06)$ & $47(36.94,57.24)$ \\
\hline
\end{tabular}

710

711 We also conducted an analysis in which the number of items on the more numerous plate $(3,4,5$, 712 6), as well as the difference in the number of items between plates (1 or 2) were entered as 713 independent variables into a log-linear analysis, alongside the Most Preferred factor as defined 714 previously (the likelihood ratio of this model was $\chi^{2}(14)=14.71, p=.40$; note that the 1 vs 2 715 condition had to be excluded from this analysis to avoid empty cells). As before, the only factor 716 retained by the model was again Most Preferred, $\left.\chi^{2}(1)=47.52, p<.001\right)$.

717 So, there is evidence here that dishes with more items on them are preferred to items with fewer 718 items. This is despite 2 key points. The first is that the portions on each plate were approximately 719 equated in terms of food (in actual fact, there was limited evidence for the fewer itemed dished 720 containing more food than its more numerous counterpart stimulus), which is bolstered by the fact 721 that hunger did not influence preferences. The second point to note is that the larger differences in 722 the number of items did not lead to more exaggerated preferences for the more numerous dish. 
723 If one considers that the dependent variable here represents the number of individuals who have

724 preference for one stimulus over another, and that we have controlled for effects such as the 725 volume of food which would likely influence participants' decisions if they were hungry, our 726 results could simply reflect individual preference (some individuals prefer plates with a lot of food 727 elements, others prefer less elements), that is unchanging over the manipulations introduced in this 728 study.

\section{GENERAL DISCUSSION}

731 Taken together, the results of the 7 experiments reported here provide no support for the 732 commonly-stated assertion that an odd number of items on a plate would be preferred to an even number. After controlling for portion size (Experiments 2-3, Supplementary Experiments A and B), testing for plate overcrowding (Experiment 2) and perceptual distortions (Experiment 4), only one group of participants were found to sometimes prefer 3-item dishes as opposed to 4 (Figure 3; Experiment 1, the Science Museum); on contrast, two further groups of participants recruited through MTurk (Experiments 2-5) and Prolific Academic (Experiment 6) preferred 4-item dishes over three. Indeed, in our final study (Experiment 7), we found that the plate with the more food items was generally preferred, over that containing fewer items. We will discuss several major issues with these findings after briefly summarising each of the experiments in turn.

\section{OVERVIEW OF THE STUDIES}

742

744

745

746

747

The results of the first experiment, conducted in collaboration with the Science Museum with 1816 participants, were ambiguous, with 3-items being preferred over 4-items when those items were vertically orientated and on a circular plate only. In all other conditions, 4-items were preferred. This was followed up with a series of experiments that, in turn, tested, and helped control for several confounds, the first of which was ensuring that the individual food items were the same size over conditions (not so in the first study).

Next, we tested whether plate overcrowding had influenced findings in the first study. Experiment 2 explored this potential confound by testing whether the ratio between plate size and the surface area covered by the food influenced the plating preference. There was no statistical evidence for 
751 such an effect, although, descriptively, effects of food liking were less strong on larger plates than 752 on regular plates, which warrants future research. Unexpectedly, 4 item dishes were preferred in 753 all experimental conditions.

754 Several further experiments tested whether the difference in portion size over conditions in 755 Experiment 1 acted to confound the results. The relative size of the 4 -item portion was reduced 756 relative to the 3-item portion in Experiment 3, and in Supplementary Experiments A and B, with 757 the general finding being that the larger the portion, the more people were likely to pick that portion 758 over a smaller portion.

759 Expectedly, and contrary to the commonly-held belief, 4-items were preferred over 3 when portion 760 sizes were equated. In Experiment 4, we tested whether there was a perceptual distortion of portion sizes such that the 4-item dish seemed greater in size than the 3-item portion, but there was no real evidence for this. This issue is explored in a complementary paper (Woods et al., in prep).

By means of a combined analysis, there was clear evidence that portion size plays a key role in deciding which plating people prefer, with larger perceived portions more likely to be chosen. Furthermore, we found that quantifying the portion sizes over experimental condition, the Science Museum study, seemed to obey this principle as well. However, whilst the rate of change of the findings over the first study versus other studies seemed equivalent (that is, portion size change tallied with liking change), the scaling at which a 4-item dish required to seem the same size as the 3-item dish differed.

770

771

772

773

774

775

776

777

778

779
One explanation for this variation was that all the stimuli used in Experiment 1 were actually smaller than those used in the subsequent studies. When we explicitly tested for this with a repeated-measures design in Experiment 5 (to get around the issue of hardware variation in online research), this issue was, however not found to influence plating preferences.

Another explanation was that population differences from which Experiment 1 participants were from (the general public in the UK mostly) and those recruited from in other studies (Mechanical Turk) led to this shift. Experiment 6 attempted to test this by recruiting from a third population (Prolific Academic) to see whether this population's preferences differed from the other two populations; these individuals though also adhered to the same portion size dish preference principle. This new sample did not really differ from the samples recruited through Mechanical 
780 Turk, but nevertheless we cannot rule out that population and/or cultural differences have indeed 781 caused the discussed difference in results. Furthermore, it seemed that plate overcrowding has a 782 different impact on plate preference for this group, than for Mechanical Turkers.

783 Finally, we demonstrated that it was the number of items on the plate, rather than whether the plate 784 had an odd or even number of items that influenced which of two plates of food our participants 785 preferred, with the majority preferring the plate containing more elements. As the dishes in this

786

787

788

789

790

791

792

793

794

795

796

797

798

799

800

801

802

803

804

805

806 study contained approximately the same amount of food this could not be due to our participants deciding on the plate that would seem most filling. The sheer difference in the number of elements between plates also failed to influence preference ratings leading us to speculate that, in this particular study, there were simply more participants who generally preferred plates containing more elements than fewer elements. Presumably then, we would expect the same study ran with those recruited from Experiment 1 to reveal the opposite trend.

\section{CAVEATS}

\section{Generalizability}

Just how generalizable are the results obtained here with the scallop stimuli? Consider that larger scallops are typically more expensive and presumably preferred by both the chef and consumer, but which likely require more effect to cut up and eat. This trend, however, does not necessarily hold for other food types. One way of answering such a question would involve surveying just how frequently different numbers of various food items appears in natural dining situations (see Michel et al., 2015a, b, for a methodology that could help elucidate this mystery).

Another consideration is just how generalizeable these findings are to the general public. Indeed, if we base the choice of 3 vs. 4 items on culturally-based aesthetic preferences (i.e., in China, 4 items are regarded as beautiful), it seems even harder to rule out a conclusion. Is this only an issue with western chefs? Note that the idea that odd items being preferred comes from chefs working in 'fine dining' restaurants, where a full meal is served over smaller portions, and hence more attention is paid to the aesthetic appeal of each portion, ruling out, to some extent, the perceived 
807 portion-size factors that seem to have been important in determining the findings reported in this 808 paper.

$809 \quad$ Experimental design

810 Here, the pairs of dishes presented to participants were mostly identical in terms of plate shape, 811 plate size and food arrangement (vertical versus polygonal), which meant that it was impossible to 812 test for interactions between these factors. The decision to go with this design was to minimise the 813 number of trials participants would have to undertake (Experiment 2 onwards) or to ensure 814 sufficient numbers of participants per group in the Science Museum study (we were delighted that 8151816 participants took part in our study and expected a smaller sample size).

816

\section{Conclusions}

818 The evidence reported in this study suggests that a plate containing more items is generally 819 preferred over a plate containing a fewer number of items, despite those plates containing the same 820 amount of food. It seems likely though that such an effect varies over populations and cultures (cf. 821 Experiment 6), though future research will be needed to verify this claim. To arrive at a clear result 822 on this topic will be challenging, as several major issues pertaining to the experimental design and 823 generalizability of the findings still need to be controlled for. Some tangential findings arose as a 824 result of teasing apart the initially promising findings in the original study performed at the Science 825 Museum. Although not significant for 3 or 4 items on a plate (Experiment 4), we report evidence 826 in a complementary article that the perceived portion size of vertical and horizontal pairs of items 827 is distorted (Woods et al., in prep). There was also suggestive evidence for the negative impact of 828 plate overcrowding on liking, but, again, this warrants further studies to be verified and properly 829 tested for.

833 ACKNOWLEDGEMENTS: We are grateful for suggestions for explanations for the results of 834 Experiment 1 by Moritz Bernoully, Virtyt Gacaferi, Ellen Jarvis, Jens Karraß, Johanna Kuenzel, 
835 Daniel Lakens, Alejandro Salgado-Montejo, Emily Snowden, Winni Theis, Catherine Transler, 836 Pepijn Vemer, Kyra Woods, and Simon Woods. We would also like to thank the hundreds of 837 Mechanical Turk and Prolific Academic participants who took part in our studies.

838

839

840

REFERENCES

841

842 Arnheim R. (1988). The power of the center: A study of composition in the visual arts. Berkeley, 843 CA: University of California Press.

844 Bajaj, D. (2013). Effect of number of food pieces on food selection and consumption in animals 845 and humans. Unpublished doctoral dissertation. Arizona: Arizona State University.

846 Benton, D. (2015). Portion size: What we know and what we need to know. Critical Reviews in 847 Food Science and Nutrition, 55, 988-1004.

848 Bouleau, C. (1980). The painter's secret geometry. New York, NY: Hacker Books.

849 Brunstrom, J. M., Rogers, P. J., Pothos, E. M., Calitri, R., \& Tapper, K. (2008). Estimating 850 everyday portion size using a 'method of constant stimuli': In a student sample, portion size is 851 predicted by gender, dietary behaviour, and hunger, but not BMI. Appetite, 51, 296-301.

852 Daneyko, O., Zavagno, D., \& Zanuttini, L. (2011). Lightness effects in Delboeuf and Ebbinghaus 853 size-contrast illusions. Perception, 40, 464-473.

854 Deroy, O., Michel, C., Piqueras-Fiszman, B., \& Spence, C. (2014). The plating manifesto (I): From 855 decoration to creation. Flavour, 3:6.

856 Deroy, O., \& Spence, C. (2014). Can you find the golden ratio in your plate? Flavour, 3:5.

857 Forde, C. G., Almiron-Roig, E., \& Brunstrom, J. M. (2015). Expected satiety: Application to 858 weight management and understanding energy selection in humans. Current Obesity Reports, 4, 859 1-10. 
860 Geier, A. B., Rozin, P., \& Doros, G. (2006). Unit bias a new heuristic that helps explain the effect 861 of portion size on food intake. Psychological Science, 17, 521-525.

862 Henrich, J., Heine, S. J., \& Norenzayan, A. (2010). The weirdest people in the world? Behavioral 863 and Brain Sciences, 33, 61-135.

864 Hollands, G. J., Shemilt, I., Marteau, T. M., Jebb, S. A., Lewis, H. B., Wei, Y., Higgins, J. P. T., $865 \&$ Ogilvie, D. (2015). Portion, package or tableware size for changing selection and consumption 866 of food, alcohol and tobacco (Review). The Cochrane Library, 9.

867 Hauser, M. D., Carey, S., \& Hauser, L. B. (2000). Spontaneous number representation in semi868 free-ranging rhesus monkeys. Proceedings of the Royal Society of London B: Biological 869 Sciences, 267, 829-833.

870 Kerameas, K., Vartanian, L. R., Herman, C. P., \& Polivy, J. (2015). The effect of portion size and 871 unit size on food intake: Unit bias or segmentation effect? Health Psychology, 34, 670-676.

872 Latto, R., Brian, D., \& Kelly, B. (2000). An oblique effect in aesthetics: Homage to Mondrian 873 (1872-1944). Perception, 29, 981-987.

874 Latto, R., \& Russell-Duff, K. (2002). An oblique effect in the selection of line orientation by 875 twentieth century painters. Empirical Studies in the Arts, 20, 49-60.

876 McClain, A. D., van den Bos, W., Matheson, D., Desai, M., McClure, S. M., \& Robinson, T. N. 877 (2014). Visual illusions and plate design: The effects of plate rim widths and rim coloring on 878 perceived food portion size. International Journal of Obesity, 38, 657-662.

879 Michel, C., Velasco, C., Fraemohs, P., \& Spence, C. (2015a). Studying the impact of plating and 880 cutlery on ratings of the food served in naturalistic dining contexts. Appetite, 90, 45-50.

881 Michel, C., Velasco, C., Gatti, E., \& Spence, C. (2014). A taste of Kandinsky: Assessing the 882 influence of the visual presentation of food on the diner's expectations and experiences. Flavour, $8833,7$.

884 Michel, C., Velasco, C., \& Spence, C. (2015b). Cutlery matters: heavy cutlery enhances diners' 885 enjoyment of the food served in a realistic dining environment. Flavour, 4:27. 
886 Michel, C., Velasco, C., Woods, A. T., \& Spence, C. (submitted). On the importance of balance to 887 the aesthetic plating of food. Appetite.

888 Michel, C., Woods, A. T., Neuhäeuser, M., Landgraf, A., \& Spence, C. (2015c). Orienting the 889 plate: Online study assesses the importance of the orientation in the plating of food. Food Quality 890 \& Preference, 44, 194-202.

891 Palmer, S. E., Schloss, K. B., \& Sammartino, J. (2013). Visual aesthetics and human 892 preference. Annual Review of Psychology, 64, 77-107.

893 Shimoyama, S. (1976). Translation of Sakuteiki: The Book of the Garden (Tokyo: Town and City 894 Planners). Attributed to Toshitsuna Tachibana, late 11th/early 12th Century.

895 Spence, C., \& Piqueras-Fiszman, B. (2014). The perfect meal: The multisensory science of food 896 and dining. Oxford, UK: Wiley-Blackwell.

897 Spence, C., Piqueras-Fiszman, B., Michel, C., \& Deroy, O. (2014). Plating manifesto (II): The art 898 and science of plating. Flavour, 3, 4.

899 Spurlock, M. (Producer/Director) (2004). Super size me [Motion Picture]. United States: The Con.

900 Styler, C., \& Lazarus, D. (2006). Working the plate: The art of food presentation. New York, NY: 901 John Wiley.

902 Surkys, T., Bertulis, A., \& Bulatov, A. (2006). Delboeuf illusion study. Medicina (Kaunas), 42, 903 673-681.

904 Uller, C., \& Lewis, J. (2009). Horses (Equus caballus) select the greater of two quantities in small 905 numerical contrasts. Animal Cognition, 12, 733-738.

906 Van Tonder, G. J., \& Lyons, M. J. (2005). Visual perception in Japanese rock garden design. 907 Axiomathes, 15, 353-371.

908 Wilkinson, L. L., Hinton, E. C., Fay, S. H., Ferriday, D., Rogers, P. J., \& Brunstrom, J. M. (2012).

909 Computer-based assessments of expected satiety predict behavioural measures of portion-size 910 selection and food intake. Appetite, 59, 933-938. 
911 Wilson, A., \& Chatterjee, A. (2005). The assessment of preference for balance: Introducing a new

912 test. Empirical Study of the Arts, 23, 165-180.

913 Yoshino, D., Kimura, A., \& Noguchi, K. (2009). Visual illusion and aesthetic preference: Some 914 common stimulus properties. Gestalt Theory, 31, 29-42.

915 Zampollo, F., Kniffin, K. M., Wansink, B., \& Shimizu, M. (2012). Food plating preferences of 916 children: The importance of presentation on desire for diversity. Acta Paediatrica, 101, 61-66.

917 Zampollo, F., Wansink, B., Kniffin, K. M., Shimuzu, M., \& Omori, A. (2012). Looks good enough

918 to eat: How food plating preferences differ across cultures and continents. Cross Cultural 919 Research, 46, 31-49.

920 Zellner, D. A. (2015). Effect of visual cues on sensory and hedonic evaluation of food. In A. Hirsch 921 (Ed.), Nutrition and chemosensation (pp. 159-174). Boca Raton, FL: CRC Press.

922 Zellner, D. A., Lankford, M., Ambrose, L., \& Locher, P. (2010). Art on the plate: Effect of balance 923 and color on attractiveness of, willingness to try and liking for food. Food Quality and Preference, $92421,575-578$.

925 Zellner, D. A., Loss, C. R., Zearfoss J., \& Remolina S. (2014). It tastes as good as it looks! The 926 effect of food presentation on liking for the flavor of food. Appetite, 77C, 31-35.

927 Zellner, D. A., Siemers, E., Teran, V., Conroy, R., Lankford, M., Agrafiotis, A., Ambrose, L., \& 928 Locher, P. (2011). Neatness counts. How plating affects liking for the taste of food. Appetite, 57, $929642-648$. 
931 Supplementary Material

932 SUPPLEMENTARY EXPERIMENT A: 4 items much smaller in terms of volume than 3

933 items

934 The original goal of this study was to scale the 4-item stimuli so that together they would be the 935 same size as the 3-item dish. However, due to a scaling error, the 4-item food elements were, in 936 fact, much smaller than the 3-item food elements. This gives us the opportunity to see whether 'the 937 table turns' as it were, that is, whether the 3-item dishes that offer a larger portion-size than the 4938 item dishes are suddenly preferred.

939

940

MATERIALS AND METHODS

941

\section{Participants}

942 One hundred participants (38 female) were recruited from Amazon's Mechanical Turk to take part 943 in the experiment in return for a payment of .35 US dollars. The participants ranged in age from

94419 to 64 years $(M=32.7$ years, $S D=10.3)$. The experiment was conducted on $9 / 06 / 2015$, from 945 16:00 GMT onwards, over a one-hour period. The participants took an average of 95 seconds (SD $946=144)$ to complete the study. All of the participants provided their informed consent prior to taking 947 part in the study.

948

949 Stimuli, Design and Procedure

950 Here, the 4-item stimuli were individually 75\% the size (along the vertical and horizontal 951 dimensions) of each of the scallops in the 3-item plate.

953 A log-linear analysis, as defined in Experiment 2, was conducted on the data from this study (the 954 final model's likelihood ratio was $\left.\chi^{2}(14)=.97, p=1\right)$. The model only retained the effect of Items, 
$955 \chi^{2}(1)=91.54, p<.001$, with 3 -item dishes (selected 534 times, or $66.75 \%$ of the time) 2.00 times 956 more likely to be preferred as the 4-items dishes (266; Fisher's exact t-test 95\% CI were $63.37 \%$ 957 and $70.01 \%)$.

958 Given that the 3-item dishes were indeed preferred over the 4-item dishes, this implies that portion 959 size plays a role in how people decide between which of two plates of food they prefer. Note the 960 absence of any effect of plate size in this study, as compared to the Experiment 2. By making the 961 portions smaller, the plates here perhaps appeared less cluttered (or did not seem over-full; see

962 Figure 9). Although we cannot rule out that it was portion size, not visual clutter that led to such

963 effects being observed in Experiment 2 (and speculated on in Experiment 1), drawing inspiration 964 from Occam's razor, the former being the simpler (and explainable) alternative perhaps makes it 965 the most likely.
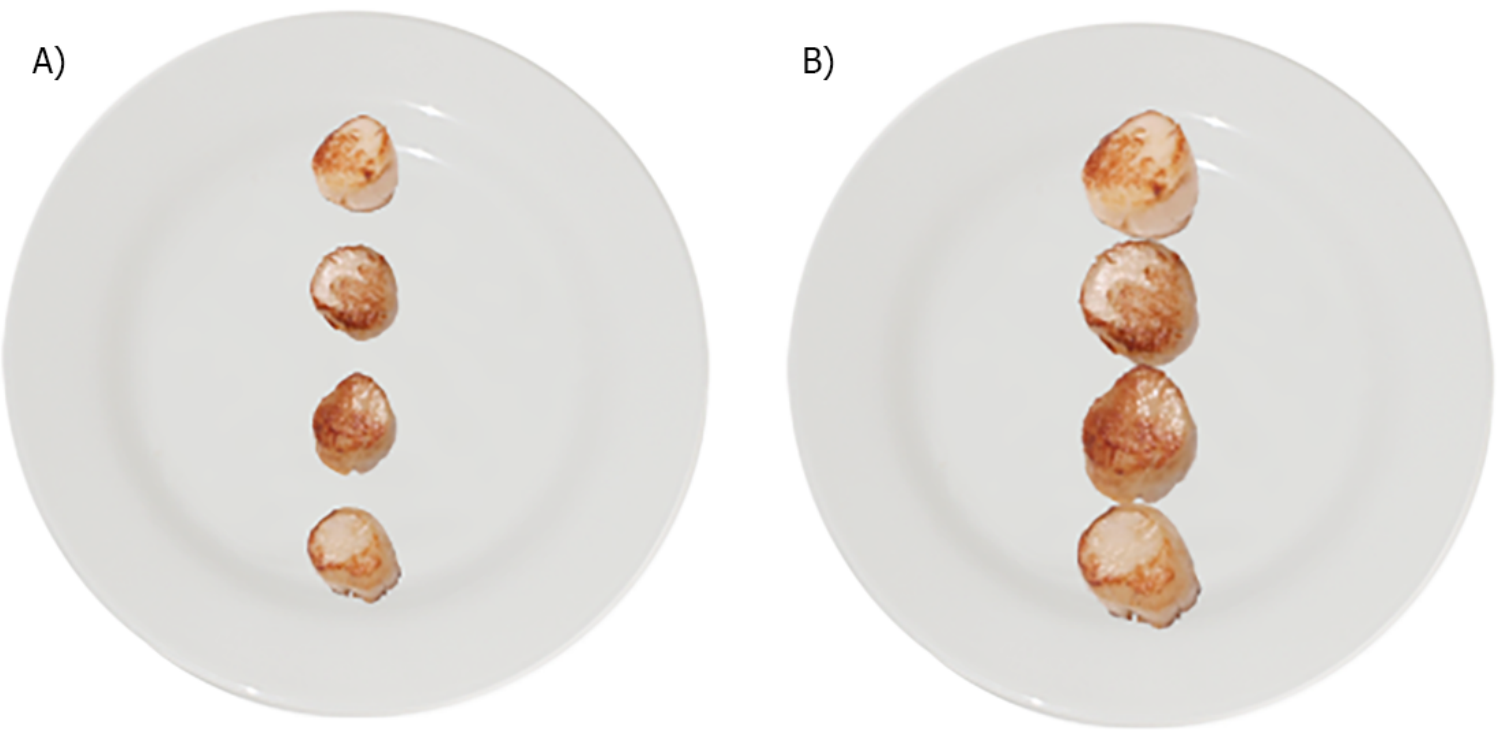

966

967 Figure 9. The vertical 4-item circular dish from Supplementary Experiment A (A) and Experiment 2 (B).

968

969 SUPPLEMENTARY EXPERIMENT B: What happens when the plates contain 970 perceptually equivalent amounts of food?

971 We expected that when the 3- and 4-item dishes were identical in terms of the perceived amount

972 of food, other influences on preference other than 'number of scallops' would be easier to detect. 
973 In order to estimate the scaling of the 4-item scallops to use, we created a linear model to predict

974 the likelihood of participants deciding upon the 3-item dish (as opposed to 4-item dish) based on

975 the scaling of 4-item scallops relative to 3-item scallops from Experiments 2-5 (we did not include

976 the data from Experiment 1, given the remaining ambiguity concerning why it differed from the

977 other experiments conducted so far; plotted in Figure 10). Using this model, the scaling to use for

978 the 4-item scallops so that participants would be equally like to decide on a 3- or 4-item dish was

979 estimated to be .84 .

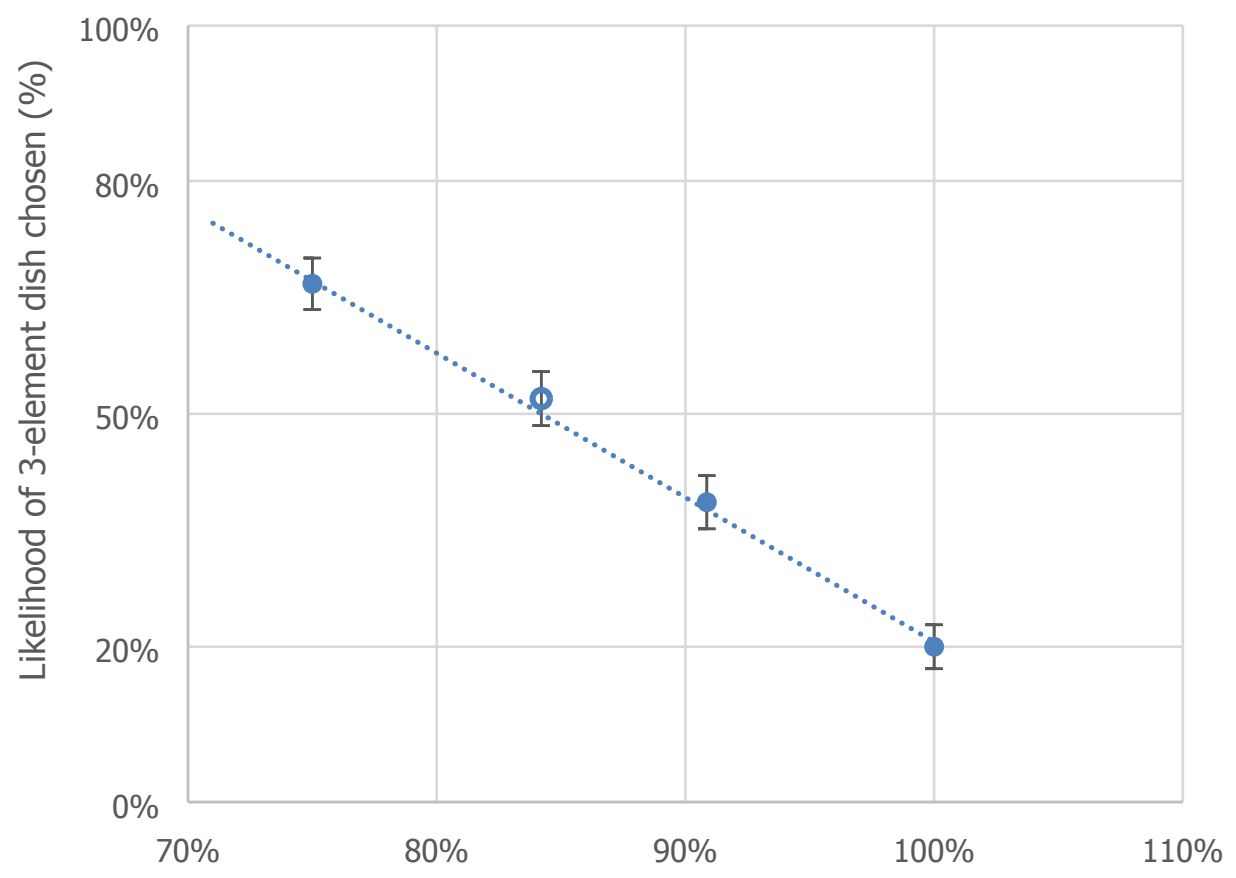

Scaling of individual 4-item scallops relative to 3-item scallops

980

981

982

983

984

985

986

987

988

989

Figure 10. Figure depicting the relationship between 4-item scallop scaling and likelihood of the 3-scallop dish being chosen, over Experiments 2 and 3, and also from the Supplementary Experiment A. The results of this study, Supplementary Experimental B is also shown (the transparent blue circle). Error bars represent the 95\% CI derived from separated Fisher's exact binomial tests.

\section{MATERIALS AND METHODS}

\section{Participants}

One hundred and two participants (34 female) were recruited from Amazon's Mechanical Turk to take part in the experiment in return for a payment of .35 US dollars. The participants ranged in 
990 age between 19 and 56 years $(M=32.7$ years, $S D=9.3)$. The experiment was conducted on 991 12/06/2015, from 16:00 GMT onwards, over a one-hour period. The participants took an average 992 of 95 seconds $(S D=109)$ to complete the study. All of the participants provided their informed 993 consent prior to taking part.

994

995 Stimuli Design and Procedure

996 These were the same as in Experiment 2 with the exception that the scaling of the food elements 997 was such that the 3-item and 4-item food portions were perceptually equal.

998 RESULTS AND DISCUSSION

999 A log-linear analysis, as defined in Experiment 2, was conducted using data from this study (the 1000 final model's likelihood ratio was $\left.\chi^{2}(15)=3.62, p=1\right)$. There were no significant effects remaining 1001 in the model and only its constant was retained. There was thus no evidence that factors other than 1002 'number of food items' played any role in dish selection in this particular study. 